\title{
Imbalances and Weakness of Hips and Thighs Muscles as One of the Sources of Low Back Pain
}

\author{
Mostafa M EL Soltan, Isra Enajeh Dow
}

\begin{abstract}
Low back pain (LBP) is a common public health problem worldwide. Musculoskeletal impairment was the most prevalent impairment in low back pain patients. Back and spine muscle impairments are the most frequently reported subcategory of musculoskeletal impairment. There is uncertainty about the source of low back pain because the number of studies of the effects of hips and thighs muscles on back pain has been few. A strong correlation between the weakness of the abdominal muscles, tightness of some hip and thigh muscles, lower physical activity and decreased endurance of hips, thighs and back muscles with low back pain. Imbalances of hips and thighs muscle strength that occurs in many years have been thought to be one of the most reasons for developing low back pain. The present study is the nature of multi-factorial cross-sectional none-experimental study. The aim of this study was to identify whither muscles weakness and imbalances of hips and thighs have a negative effect and developing low back pain. Twenty fife LBP patients has subscripted in this study from mixed gender their ages ranged from 20 to 70 years. Results suggest that hips and thighs muscle groups is extremely weak and do not match the test value of $5(P=0.00$. $)$, therefore hi level imbalances between hips muscles and thighs muscle were confirmed in this study, right hip extension versus left hip extension ( $P=0.001$ ), right hip flexion versus left hip flexion ( $P$ $=0.05)$, right hip flexion versus right hip extension $(P=0.000)$, Right Knee extension versus Right Knee flexion $(P=0.009)$, left hip flexion versus left hip extension ( $P=(0.007)$, left Knee extension versus left Knee flexion ( $P=0.043$ ).
\end{abstract}

Index Terms - Muscle Weakness, Imbalances, Low Back Pain ,Hip and Thigh Muscles.

\section{INTRODUCTION}

\section{A. Low back pain}

Low back pain (LBP) is a common public health problem world-wide ( Rainer Freynhagen 2006 ). For quite a number of patients affected and developed of low back pain, such disorder is extremely disabling. indisputably, the disease is associated with a serious risk of ending in early retirement or an inability to cope with activities of daily living. Low back pain was recently reported to be the single largest cause of disability across the globe ( NicholasA.Coopr 2015 ). In USA many Americans have experienced low back pain, and reported having low back pain lasting at least 1 day in the past 3 months, which suspected the most common cause of daily activity limitation in population less than 45 years, the second

Mostafa M El Soltan, Physiotherapy Department, University of Zawia/ Faculty of Medical Technology/ Libyan ministry of thigh education and researches, Zawia City, Libya

Isra Enajeh Dow, Physiotherapy Department, University of Zawia/ Faculty of Medical Technology/ Libyan ministry of thigh education and researches, Zawia City, Libya most frequent reason for visits care centers, the fifth-ranking cause of admission to hospital. Surgical procedures classified as the third most common procedures to treat LBP and disc herniation. In the UK low back pain was the largest single cause of absence from work in 1988-89. many researchers certify to the high frequency of back complaints in society. The patho-physiological mechanisms of pain generation in back pain are complex, diagnostic tools for somewhat do not reach the accurate cause and patients' needs in many respects. However, there is a huge unanimity among many disciplines involved in the cause of back pain that the classification of LBP disorders has to be based on the underlying mechanism, in order to describe a clear treatment plan, recommendations and to ensure appropriate management for individual patient. The causes of low back pain should be correctly diagnosed, muscles weakness and insufficiency of pelvic ligaments could contribute to overload and pain during activities with regard to muscular influences on LBP. Hips and thighs muscles play a significant role in transferring forces from the lower extremity up toward the spine during upright activities and thus theoretically may influence the development of LBP. Delayed firing and poor endurance of gluteus maximus and gluteus medius muscles have been noted in individuals with low back pain and lower extremities instability. (Scot F.Nadlr 2001). DeVita et al. noted an alteration in firing of the proximal hips musculature in those with anterior cruciate insufficiency. Jaramillo et al, demonstrated significant strength deficits of the ipsilateral gluteus medius in patients who had undergone knee surgery (Scot F.Nadlr 2001) (Jarmaillo .J. 1994). In a study of back and hips extensor muscle fatigability in chronic low back pain patients Kankaanpaa and Leinonen demonstrated an obvious poor endurance of the gluteus maximus in those with chronic LBP. Recently, many researches applying lumbar segmental stabilization exercise to chronic low back pain patients is being conducted. Nonetheless, researches focusing on the muscles of the hips and thighs is insufficient, although there are clear associations between low back pain and hips thighs muscles positively affect low back pain. Reiman has observed that functional disorder resulting from weakening of the hips muscles and restricted hips joints range of motion has elements related to pathology of the waist and the lower extremity. There is a biodynamic relationship between low back pain, hips joints, and hips muscles, when applying an exercises program for chronic low back pain patients. Stability of the pelvis and hips joints and strengthening of the hips muscles are important. Their results showed that hips muscles strengthening exercises in addition to lumbar segmental stabilization exercises increased the stability of the 
hips joints and pelvis, which was conducive to increasing lumbar segmental stability. Despite its detrimental association with social and work-related activities, the precise causes and the mechanisms of LBP has not yet been determined. However, by using different designs and measurement procedures, argumentative results have been noted in the literature the major theory has long speculated that changes in muscles strength are the main cause of LBP.

\section{B. Muscles weakness}

Current interdisciplinary practice guidelines show strong evidence for exercise as an intervention for LBP and thus recommend exercises for management of both acute and chronic LBP to strength and avoid the weakness of back, hips and pelvic area (Nicholas A. Cooper 2015). A variety of exercises interventions have been studied and have been shown to improve pain and disability in individuals with LBP. Most patients who have chronic low back pain are matched to exercises treatment based on physical and mechanical assessment and evaluation. Travell and Simons give an obvious description of myofascial pain from the gluteus medius muscle as a common component of low back pain. lately, Njoo and van der Does has reported a higher prevalence of gluteus medius myofascial trigger points pain in patients have chronic lumbar spine pain. in 2015 Nicholas A. and Cooper suggested that gluteus medius weakness is associated with myofascial pain. Muscle neuromuscular activation in the pelvic region plays a primary role for the physiologic interaction and coordination of pelvis, spine and lower limbs movements in human. By looking at the stabilization concept Panjabi (1992a,b; Norris 1995), has stated, disturbances of the musculoskeletal and fascial system can be prerequisites for or also consequences of pathological of lumbar spine syndrome. In a large samples study of 370 children aged 11-17 years Salminen advertises a significant correlation between low back pain and weakness of the abdominal muscles and tightness of the hamstring muscles ( $\mathrm{P}$ $<0.01)$. It is possible that muscle weakness is a long-term side effect of rest. The theoretic influence of muscle force on stability of the pelvis is reported in the literature. Newcomer and Sinaki did not find any correlation between LBP and a low frequency of physical activity. However, the cumulated prevalence of LBP was correlated with a high level of physical activity. Taimela et al. found a similar association between LBP and high level of physical activity in a sample of 1171 schoolchildren aged 7-16 years. This gives us an important indication according to the previous discussion that high level activities may use a group of muscles more than others making muscles imbalances.

\section{Muscles imbalances and asymmetry}

Imbalances of hip muscles strength that accurse in many years by using one or two muscle group more than others has been thought to be one of the most reason for increasing back pain. asymmetry of the strength of hip abductors has been correlated with increased probability to seek care for LBP in athletes. Hip abductor and adductor muscles play a significant role in pelvic lateral stability. Any symmetry changes or imbalance in the function of the previous mentioned muscles and due to muscle shortening or weakness may cause pelvic obliquity and functional lumbar lateral bending. Furthermore, considering the role of these muscles in sacroiliac joint stability any disturbance in their function can lead to sacroiliac joint instability and LBP. However, the negative effects of weakness or shortening of these muscles on LBP have not been directly assessed. Recently Nelson-Wong and colleagues declared that subjects who developed LBP during an experimental standing task had a different recruitment pattern of gluteus medius muscle compared to those who did not develop LBP. When imbalances between the abdominal muscles and trunk extensor muscles occur this imbalances will make a negative effect on low back pain and reduces stabilization of the lumbar segment. In addition, low back pain patients experience a decrease in trunk and hip activities because of "pain, structural damage, and inhibition of the reflex muscle contraction mechanism" and due to decrease of the activity and unused of some of trunk and hips muscles for a long time, muscles atrophy and a decrease in muscles strength and endurance occur, which aggravate low back pain and bring about secondary lumbar segment damage and physical disability (Cheol Jeong 2015). Thighs and hips muscles, which consist of stabilizer muscles with lumbar spine muscles play a significant role in lumbar stability and as a basic support for pelvic, lumbar segmental stabilization, strengthening of superficial and deep stabilizer muscles and co-coordination are necessary. As a function of the gluteus maximus muscle, sacroiliac joints deliver loads from the trunk to the lower limbs however, if the movement of this joints excessively occur will result in pressure on the joints and discs between the L5-S1 vertebral body, sacroiliac joint, and pubic symphysis, which leads to functional failure of the sacroiliac joint and low back pain. Low back pain patients have a decreased balance ability compared with individuals not affected with such disease. When LPB patients are exposed to an unexpected load the muscles should swiftly respond to maintain balance and posture against the loads, however, patients of chronic back pain have a delayed response time, triggering a problem in postural maintenance and balance. In a study has been conducted in 2002 on hip muscles imbalance and low back pain in athletes:( influence of core strengthening) Nadler et al reported that "the core program however, seems to have had a role in modifying hip extensor strength balance". Nadler et al. have found a significant asymmetry in hip extensor strength in female athletes who have experience of LBP. Athletes' female with a report of LBP in the last year were observed to have significant weakness of right as compared with left hip extensors.

\section{RESULTS}

Table I

This table showing the cases which subscribed in this study, as the table show females were ten LBP patients and males were fifteen.

\begin{tabular}{|c|c|c|c|c|}
\hline & & Frequency & Percent & $\begin{array}{l}\text { Valid } \\
\text { Percent }\end{array}$ \\
\hline \multirow[t]{3}{*}{ Valid } & males & 15 & 60.0 & 60.0 \\
\hline & females & 10 & 40.0 & 40.0 \\
\hline & Total & 25 & 100.0 & 100.0 \\
\hline
\end{tabular}


Table II

This table shows the mean of strength of hips and thighs muscles groups in our population.

\begin{tabular}{ll|l|l|l} 
One-Sample Statistics & \multicolumn{2}{l}{} & & Std. Error Mean \\
\hline Right hip flexion muscles group & $\mathrm{N}$ & Mean & Std. Deviation & .14514 \\
\hline Right hip extension muscles group & 25 & 3.1200 & .72572 & .17321 \\
\hline Right hip adduction muscles group & 25 & 2.2000 & .86603 & .18903 \\
\hline Right hip abduction muscles group & 25 & 3.3200 & .94516 & .17010 \\
\hline Right hip internal rotation muscles group & 25 & 3.1600 & .85049 & .14514 \\
\hline Right hip external rotation muscles group & 25 & 3.1200 & .72572 & .14967 \\
\hline Right Knee extension muscles group & 25 & 3.3200 & .74833 & .14283 \\
\hline Right Knee flexion muscles group & 25 & 3.5200 & .71414 & .14922 \\
\hline Left hip flexion muscles group & 25 & 3.1600 & .74610 & .10000 \\
\hline Left hip extension muscles group & 25 & 3.4000 & .50000 & .21229 \\
\hline Left hip adduction muscles group & 25 & 2.7200 & 1.06145 & .19596 \\
\hline Left hip abduction muscles group & 25 & 3.2800 & .97980 & .08165 \\
\hline Left hip external rotation muscles group & 25 & 3.2000 & .40825 & .15790 \\
\hline Left hip internal rotation muscles group & 25 & 2.9600 & .78951 & .18547 \\
\hline Left Knee extension muscles group & 25 & 3.1200 & .92736 & .17436 \\
\hline Left Knee flexion muscles group & 25 & 3.5200 & .87178 & .18257 \\
\hline
\end{tabular}

Table III

This table shows the strength of each muscles groups in both hips and thighs segments.

\section{One-Sample Test}

Test Value $=5$

\begin{tabular}{|c|c|c|}
\hline & $\mathrm{T}$ & $\begin{array}{l}\text { Sig. } \\
\text { tailed })\end{array}$ \\
\hline Right hip flexion muscles group & $-12.953-$ & .000 \\
\hline $\begin{array}{llll}\text { Right } & \text { hip extension muscles } \\
\text { group } & & & \end{array}$ & $-16.166-$ & .000 \\
\hline $\begin{array}{l}\text { Right hip adduction muscles } \\
\text { group }\end{array}$ & $-8.887-$ & .000 \\
\hline $\begin{array}{l}\text { Right hip abduction muscles } \\
\text { group }\end{array}$ & $-10.817-$ & .000 \\
\hline $\begin{array}{lll}\text { Right hip internal } & \text { rotation } \\
\text { muscles group } & & \end{array}$ & $-12.953-$ & .000 \\
\hline $\begin{array}{lll}\text { Right hip external } & \text { rotation } \\
\text { muscles group } & & \end{array}$ & $-11.225-$ & .000 \\
\hline $\begin{array}{l}\text { Right Knee extension muscles } \\
\text { group }\end{array}$ & $-10.362-$ & .000 \\
\hline $\begin{array}{llll}\text { Right } & \text { Knee } & \text { flexion } & \text { muscles } \\
\text { group } & & & \end{array}$ & $-12.331-$ & .000 \\
\hline Left hip flexion muscles group & $-16.000-$ & .000 \\
\hline Left hip extension muscles group & $-10.740-$ & .000 \\
\hline $\begin{array}{l}\text { Left hip adduction muscles } \\
\text { group }\end{array}$ & $-8.777-$ & .000 \\
\hline $\begin{array}{llll}\text { Left } & \text { hip } & \text { abduction } & \text { muscles } \\
\text { group } & & & \end{array}$ & $-22.045-$ & .000 \\
\hline $\begin{array}{lll}\text { Left hip external rotation } \\
\text { muscles group }\end{array}$ & $-12.919-$ & .000 \\
\hline $\begin{array}{lll}\text { Left hip internal } & \text { rotation } \\
\text { muscles group } & & \\
\end{array}$ & $-10.136-$ & .000 \\
\hline $\begin{array}{llll}\text { Left } & \text { Knee extension muscles } \\
\text { group } & & & \end{array}$ & $-8.488-$ & .000 \\
\hline Left Knee flexion muscles group & $-9.859-$ & .000 \\
\hline
\end{tabular}

Table IV

This table shows the imbalances of muscle strength in both sides of the hips and knees muscles groups.

\section{Paired Samples Test}

Paired Differences

Sig.

$\mathrm{T}$ (2-tailed)

\begin{tabular}{|ll|r|r|}
\hline Pair 1 & Right hip flexion - Left hip flexion & $-2.064-$ & .050 \\
\hline Pair 2 & Right hip extension - Left hip extension & $-3.641-$ & .001 \\
\hline Pair 3 & Right hip adduction - Left hip adduction & .214 & .832 \\
\hline Pair 4 & Right hip abduction - Left hip abduction & $-.253-$ & .802 \\
\hline Pair 5 & $\begin{array}{l}\text { Right hip external rotation - Left hip } \\
\text { external rotation }\end{array}$ & 1.445 & .161 \\
\hline Pair 6 & $\begin{array}{l}\text { Right hip internal rotation - Left hip } \\
\text { internal rotation }\end{array}$ & 1.155 & .260 \\
\hline Pair 7 & $\begin{array}{l}\text { Right Knee extension - Left Knee } \\
\text { extension }\end{array}$ & .000 & 1.000 \\
\hline Pair 8 & Right Knee flexion - Left Knee flexion & $-.238-$ & .814 \\
\hline
\end{tabular}


Table V

This table shows the imbalance between muscle strength in the same side (anterior \&posterior muscles groups ) of right hip and thigh muscles group.

\section{Paired Samples Test}

\begin{tabular}{|c|c|c|c|}
\hline & & $\begin{array}{l}\text { Paired } \\
\text { Differen }\end{array}$ & \\
\hline & & $\mathrm{T}$ & Sig. (2-tailed) \\
\hline Pair 1 & $\begin{array}{l}\text { Right hip flexion - Right } \\
\text { hip extension }\end{array}$ & 4.433 & .000 \\
\hline Pair 2 & $\begin{array}{l}\text { Right hip adduction - } \\
\text { Right hip abduction }\end{array}$ & .700 & .491 \\
\hline Pair 3 & $\begin{array}{l}\text { Right hip internal rotate } \\
\text { - Right hip external } \\
\text { rotation }\end{array}$ & -1.549 & .134 \\
\hline Pair 4 & $\begin{array}{l}\text { Right Knee extension - } \\
\text { Right Knee flexion }\end{array}$ & 2.823 & .009 \\
\hline
\end{tabular}

\section{Table VI}

This table shows the imbalance between muscle strength in the same side (anterior \&posterior muscles groups ) of left hip and thigh muscles group.

\section{Paired Samples Test}

\begin{tabular}{|c|c|c|c|}
\hline & & Paired I & differences \\
\hline & & $\mathrm{T}$ & Sig. (2-tailed) \\
\hline Pair 1 & $\begin{array}{l}\text { Left hip flexion - Left hip } \\
\text { extension }\end{array}$ & 2.971 & .007 \\
\hline Pair 2 & $\begin{array}{l}\text { Left hip adduction - Left } \\
\text { hip abduction }\end{array}$ & .401 & .692 \\
\hline Pair 3 & $\begin{array}{l}\text { Left hip external rotation - } \\
\text { Left hip internal rotation }\end{array}$ & $-\overline{1}-000-$ & .327 \\
\hline Pair 4 & $\begin{array}{l}\text { Left Knee extension - Left } \\
\text { Knee flexion }\end{array}$ & 2.138 & .043 \\
\hline
\end{tabular}

\section{DISCUSSION}

The main goal of this study was exploring the relationship between low back pain, weakness and imbalances of muscles groups in both sides of the hips and thighs muscles. Based on anatomical position and function of the hip muscles in coordination and stability in pelvic region, any damage or imbalances in these muscles lead to LBP. In line with our expectation, muscles groups of the hips and thighs (both sides left \& right) are weaknesses and less than 5 degree, results of this study showed that hips and thighs muscles groups are very weak, this may be the main reason sources of LBP. By looking to the table ( 3 ) this table shows the difference of the strength of each muscles groups with the test value of 5 which has been classified as the maximum grade of any muscle strength, as the table shows the $\mathrm{P}$ value of the one sample $\mathrm{T}$ test for each hips and thighs muscles groups are< $0.05,(\mathrm{P}=0.00)$ this supports our hypothesis which said that muscles strength of the hips and thighs muscles groups are less than 5 degree for all our target population. Muscles strength imbalances of hips muscles groups have been also investigated as possible causes of low back pain. Recently, many studies reported that patients with low back pain have a decreased balance ability of musculature compared with healthy people ( Cheol 'Jeong 2015), patient with low back pain often avoid using some of their muscles with wrong position in life activities because fear of pain, this leads to overcome of the used muscle upon un-used muscles which later leads to imbalances in hips and thighs muscles. Imbalances which being confirmed between right hip flexion and left hip flexion, right hip extension and left hip extension may one the impairments lead to low back pain. Table ( 4 ) appears a significant differences between right hip flexion and left hip flexion( $\mathrm{p}$ value $=0.050$ ) some studies have shown decreased iliopsoas muscle length and strength in patients with LBP because the iliopsoas muscle attaches to the pelvis and lumbar spine, some have assumed that tightness of these muscles causes increases lumbar lordosis which, in turn, can result in LBP ( Mohammad Reza 2002 ). The same table shows a significant differences between right hip extension and left hip extension ( $\mathrm{p}$ value $=0.001$ ) Several studies have reported a significant decrease in back and hip extensor muscles endurance in patients with LBP. Current theory suggests that the proper way to perform a lifting task is to lift from a squatted position and flex the hips and knees while minimizing flexion of the lumbar spine. When lumbar flexion predominates in a lifting task, the risk for injury and pain is increased. To lift from a squatted position, an individual must have adequate strength and endurance of the gluteus maximus and quadriceps muscle groups in particular. If the gluteus maximus and quadriceps are deconditioned correct lifting may not be performed and the risk for recurrence of LBP may be increased. The gluteus maximus in particular is often weak in individuals with chronic LBP and has been found to be more fatigable than those in healthy individuals. (Kankaanpaa, M., Taimela, S., Laaksonen, D., et al. (1998). Decreased back and hips muscles endurance causes muscular fatigue and overloads soft tissue and passive structures of the lumbar spine resulting LBP. In the another hand, although there is a very weaknesses in the both side of the hips thighs regions, however there is no significant differences between these groups, right knee flexion versus left knee flexion, right knee extension versus left knee extension, right hip external rotation versus left hip external rotation, right hip internal rotation versus left hip internal rotation, right hip abduction versus left hip abduction and hip adduction versus left hip adduction the $P$ value of the paired sample test is $.81,1.00, .16, .26, .80, .83$ respectively. When imbalances between the abdominal muscles of the trunk and extensor muscles occurs, it triggers low back and reduces stabilization of the lumbar segment (Cheol Jeong 2015 ). In our hypothesis which expected that imbalances between muscle power of the anterior and posterior will increase LBP, the results of this study shows strength imbalances in some groups of hip and thigh muscles in one side By looking to the table ( 5 ) this table shows imbalances between muscles strength in the right side. Statistically, there is a significant difference between right hip flexion and right 
hip extension ( $\mathrm{p}$-value $=0.000)$. moreover, a significant difference has been found between right knee flexion versus right knee extension ( $\mathrm{p}$-value $=0.009$ ). However as we maintained in the previous discussion there is no differences between some muscles groups in the hips region in both sides we can also confirm that there is insignificant differences between some muscles groups in the hip region in one side ( right side ), right hip abduction versus right hip adduction ( $\mathrm{p}$ value $=0.491)$, right hip internal rotation versus right hip external rotation $(\mathrm{p}$ value $=0.134)$.

In the left side of the muscles groups, table ( 6 ) shows imbalance between muscle strength in hip and thigh muscle groups, a significant differences has been found between left hip flexion versus left hip extension $(p$ value $=0.007$ ) furthermore, there is significant differences between left knee flexion and left knee extension ( $p$ value $=0.043$ ). from this results one can say that the most impairment strength imbalances are in hip extension versus flexion and knee flexion versus knee extension of one side which being confirmed in both side, this muscles groups imbalances will effect stability of pelvic region which subsequently effect the lumber spin region. Hamstrings tightness is one of the most common findings in patients with LBP, It is thought that, due to the attachments of hamstrings to the ischial tuberosity, hamstrings tightness generates posterior pelvic tilt and decreases lumbar lordosis, which can result in LBP ( Mohammad Reza 2002 ). In contrast, no differences between left hip abduction and left hip adduction $(\mathrm{p}$ value $=0.692)$ and also insignificant difference in left side external rotation with left side internal rotation $(\mathrm{P}=32)$. There is no differences between left hip internal rotation and left hip external rotation $(\mathrm{p}$ value $=0.327)$.

\section{CONCLUSION}

Through the application a number of statistical tests on some of LBP patients to study the relationship between muscle weakness and low back pain, and effect of muscle weakness and imbalances on the hips and thighs muscles groups, we conclude that the muscle power of hips and thighs muscles groups is less than degree of fife, in addition to imbalance in muscles strength in the hips and thighs in both sides. Moreover, the imbalances which have been found between the posterior and anterior muscle groups on one side maybe also the mean source reason for developing and increasing low back pain. The most affected muscles are gluteus maximum muscle, rectus femoris muscle, psoas major muscle, quadriceps muscle group and hamstring muscle group. in addition, Imbalances and weakness which have been found in this study could be the main source of low back pain. Finally we recommend that strength exercises program should be given to individuals sever of low back pain to prevent any weakness or imbalances may occur in this muscles groups.

\section{REFERENCES}

[1] A. McGregora, D. Hukinsb, 2009. Lower limb involvement in spinal function and low back pain. Journal of Back and Musculoskeletal Rehabilitation.

[2] D. College, B. Joseph, M. Kevin. 2010. Anatomy and biomechanics of the hip.

[3] F. Balagué (1999): Non-specific low back pain in children and adolescents: risk factors.

[4] F. Michael, (2000). Hip abductor weakness in distance runners with iliotibial band syndrome.

[5] F. Scott, (2002). Hip muscle imbalance and low back pain in athletes: influence of core strengthening. Department of Physical Medicine and Rehabilitation, UMDNJ-NJ Medical School, Newark, NJ; Med. Sci. Sports Exerc., Vol. 34, No. 1, 2002, pp. 9-16.

[6] F. Natasha, W. Stephanie, N. Helen (2011). A review of the anatomy of the hip abductor muscles, gluteus medius, gluteus minimus, and tensor fascia lata.

[7] F. Leung, M. Mendis, W. Stanton, J. Hides. 2015. The relationship between the piriformis muscle, low back pain, lower limb injuries and motor control training among elite football players.

[8] W. Hodges, A. Richardson.1996. Inefficient muscular stabilization of the lumbar spine associated with low back pain. Spine, 21:2640-2649.

[9] J. Jaramillo, W. Worrell, D Ingesrol. 1994 . Hip isometric strength following knee surgery. Orthopedic sport physiotherapy, 20, 160, 165.

[10] C. Jeong. 2015. The effects of gluteus muscle strengthening exercise and lumbar stabilization exercise on lumbar muscle strength and balance in chronic low back pain patients. J. Phys. Ther. Sci.27: 3813-3816.

[11] J. Cheol, S. Jae, K. cheol, N. chan. 2015. The effects of gluteus muscles strengthening exercises and lumber stabilization exercises on lumber muscles strength and balance in chronic low back pain patients. Republic of Korea.

[12] J. salmine, B. Troussier, F. Balage 1999. Non- specific low back pain in children and adolescents: risk factors.

[13] D. Karen. 2010. The Relationship Between Hip-Abductor Strength and the Magnitude of Pelvic drop in Patients with low back pain.

[14] L. Danneels. 2001. Effects of three different training modalities on the cross sectional area of the lumbar multifidus muscle in patients with chronic low back pain.

[15] M. Nourbakhash, PT, PhD (2002). Relationship between mechanical factorsand incidence of low back pain J Orthop Sports Phys Ther2002;32:447-460.

[16] E. MacIntosh, N. Bogduk. 1996. The anatomy and function of the lumbar back muscles. In Boyling, J.D., and Palastanga, N. (eds.): Grieve's Modern Manual Therapy. The Vertebral Column, 2nd ed. New York, Churchill Livingstone, pp. 189-209.

[17] M. Kankaanpaa, S. Taimela, D. Laaksonen, et al. 1998. Back and hip extensor muscle fatigability in chronic low back pain patients and controls. Arch. Phys. Med. Rehabil., 79:412-418.

[18] M. McGill. 1998. Low back exercises: Evidence for improving exercise regimens. Phys. Ther., 78:754-766.

[19] M. Panjabi. 1992. The stabilization system of the spine. Part I, dysfunction, adaptation and enhancement spinal disorders. 5: 383-393, 397.

[20] N. Mohammad. 2002. Relationship between mechanical factors and incidence of low back pain .

[21] M. Nalder, Melissa prince. T, P, Stitik. Joseph, H, Feinbery. Philadelphia 2000. The relationship between lower back pain and hip muscle strength in male and female collegiate athletes.

[22] A. Nicholas, B. Springer. 2015. Prevalence of gluteus medius weakness in people with chronic low back pain compared to healthy controls.

[23] K. Njoo, E. Does. 1994. The occurrence and inter-rater reliability of myofascial trigger points in the quadratus lumborum and gluteus medius: a prospective study in non-specific low back pain patients and controls in general practice. Department of Family Medicine, Erasmus University Rotterdam, The Netherlands.

[24] R. Freynhagen, R. Baron, U Gockel, 2006. a new screening questionnaire to detect neuropathic components in patients with back pain. Curr Med Res.

[25] T. Kleinig, B. Brophy, C. Maher 2011. Back pain and leg weakness MJA; 195: 454-457. 